\title{
A SUFFICIENT CONDITION FOR LINEAR GROWTH OF VARIANCES IN A STATIONARY RANDOM SEQUENCE
}

\author{
RICHARD C. BRADLEY, JR.
}

\begin{abstract}
Suppose $\left(X_{k}, k=\ldots,-1,0,1, \ldots\right)$ is a weakly stationary random sequence. For each positive integer $n$ let $S_{n} \equiv X_{1}+\cdots+X_{n}$ and let $\tau(n)=$ $\operatorname{Sup}\left\{\left|\operatorname{Corr}\left(\Sigma_{k-1}^{0} X_{k}, \Sigma_{k=m}^{m+I} X_{k}\right)\right|: m>n, I>0\right\}$. If $\operatorname{Var} S_{n} \rightarrow \infty$ as $n \rightarrow \infty$ and $\sum_{n=0}^{\infty} \tau\left(2^{n}\right)<\infty$, then $n^{-1} \operatorname{Var} S_{n}$ converges to a finite positive limit as $n \rightarrow \infty$. A bound on the rate of convergence is obtained.
\end{abstract}

Let $\left(X_{k}, k=\ldots,-1,0,1, \ldots\right)$ be a weakly stationary sequence of random variables on a probability space $(\Omega, \mathcal{F}, P)$. That is, $E X_{k}^{2}<\infty \forall k$, there are constants $\mu$ and $\gamma(0)$ such that $E X_{k}=\mu$ and $\operatorname{Var} X_{k}=\gamma(0) \forall k$, and there is a sequence of numbers $\gamma(1), \gamma(2), \gamma(3), \ldots$ such that $\operatorname{Cov}\left(X_{k}, X_{l}\right)=\gamma(|k-l|)$ $\forall k \neq l$. For $-\infty \leqslant J \leqslant L \leqslant \infty$ let $H_{J}^{L}$ denote the $L^{2}$-closure of the linear space spanned by the r.v.'s $X_{k}-\mu, J \leqslant k \leqslant L$. For $n=1,2,3, \ldots$ let $S_{n}=X_{1}+X_{2}$ $+\cdots+X_{n}$ and define the quantities

$$
\begin{aligned}
& r(n)=\operatorname{Sup}\left\{|\operatorname{Corr}(f, g)|: f \in H_{-\infty}^{0}, g \in H_{n}^{\infty}\right\}, \\
& \tau(n)=\operatorname{Sup}_{\substack{m>n \\
I>0}}\left|\operatorname{Corr}\left(\sum_{k=-I}^{0} X_{k}, \sum_{k=m}^{m+I} X_{k}\right)\right| .
\end{aligned}
$$

Obviously the sequences $\{r(n)\}$ and $\{\tau(n)\}$ are each nonincreasing, and $0 \leqslant \tau(n)$ $\leqslant r(n)$.

Ibragimov and Rozanov [3, Note 2, p. 190] proved the following theorem (it was originally proved in [4]).

TheOREM 0 (IBRAgIMOV AND ROZANOv). If $\left(X_{k}\right)$ is weakly stationary and $\sum_{n=0}^{\infty} r\left(2^{n}\right)<\infty$, then $\left(X_{k}\right)$ has an absolutely continuous spectral distribution function, with a continuous spectral density $f(\lambda)$.

In [3, Note 2, p. 190] this is stated for stationary Gaussian sequences, but the extension to general weakly stationary sequences is easy. Ibragimov [2, Theorem 2.2] proved a central limit theorem for strictly stationary random sequences satisfying a condition similar to but stronger than $\Sigma r\left(2^{n}\right)<\infty$; in that theorem it was assumed that $f(0)>0$. In central limit theorems for weakly dependent random variables it is common practice to assume $\operatorname{Var} S_{n} \rightarrow \infty$ as $n \rightarrow \infty$. Consider the question whether $f(0)>0$ follows if one assumes $\Sigma r\left(2^{n}\right)<\infty$ and $\operatorname{Var} S_{n} \rightarrow \infty$.

Received by the editors November 26, 1980 and, in revised form, February 25, 1981.

1980 Mathematics Subject Classification. Primary 60G10; Secondary 60G99.

Key words and phrases. Weakly stationary, variance of partial sums, spectral density. 
The answer is affirmative, and one can prove this as a corollary to Theorem 0 by using the results in Chapters 4 and 5 of [3]. It also follows easily from the following theorem.

THEOREM 1. If $\left(X_{k}\right)$ is weakly stationary, $\operatorname{Var} S_{n} \rightarrow \infty$ as $n \rightarrow \infty$, and $\sum_{n=0}^{\infty} \tau\left(2^{n}\right)$ $<\infty$, then the following statements hold:

(i) There exists a finite positive constant $\sigma^{2}$ such that $\operatorname{Lim}_{n \rightarrow \infty} n^{-1} \operatorname{Var} S_{n}=\sigma^{2}$.

(ii) For each $\gamma, 0<\gamma<1$, there exist positive constants $C$ and $D$ such that for all $m \geqslant 1$,

$$
\left|\sigma^{2}-m^{-1} \operatorname{Var} S_{m}\right| \leqslant C\left(m^{(\gamma-1) / 2}+\sum_{n=0}^{\infty} \tau\left(\left[\left(2^{n} m\right)^{\gamma} / D\right]+1\right)\right)
$$

where $[t]$ denotes the greatest integer $\leqslant t$.

Theorem 1 covers the case where $\tau(n)=o\left((\log n)^{-(1+e)}\right)$ for some $\varepsilon>0$. For the stationary Gaussian sequence given in Example 1 on pp. 179-180 of [3], $r(n)=$ $O\left((\log n)^{-1}\right)$ and $n^{-1} \operatorname{Var} S_{n} \rightarrow \infty$ as $n \rightarrow \infty$. Berkes and Philipp [1, Theorem 4] prove an "almost sure invariance principle" for strictly stationary random sequences satisfying the $\phi$-mixing condition with a rather slow (logarithmic) mixing rate. Their conditions satisfy the hypothesis of Theorem 1 (see [1, Lemma 4.1.1, p. 44]), and it would be interesting to see if one can choose $a_{N}=\sigma^{2} N$ in the conclusion of their theorem. In the cases where $\tau(n) \rightarrow 0$ rapidly, other methods generally give much better bounds on $\left|\sigma^{2}-m^{-1} \operatorname{Var} S_{m}\right|$ than Theorem 1(ii) does.

Proof of Theorem 1. We will assume $E X_{k}=0$ and $\operatorname{Var} X_{k}=1$. Let $S_{0} \equiv 0$. For any r.v. $X$ let $\|x\| \equiv\left(E X^{2}\right)^{1 / 2}$. For each $n=1,2,3, \ldots$ let $g(n) \equiv n^{-1 / 2}\left\|S_{n}\right\|$.

LEMMA 1. If $N \geqslant 1, K \geqslant 0$, and $\tau(K+1)<\frac{1}{2}$, then for $L=2 N$ and for $L=2 N$ +1 one has

$$
\begin{aligned}
\left\|S_{N}\right\| \cdot[2(1-\tau(K+1))]^{1 / 2}\left(1-2 b /\left\|S_{N}\right\|\right) & <\left\|S_{L}\right\| \\
& \leqslant\left\|S_{N}\right\| \cdot[2(1+\tau(K+1))]^{1 / 2}\left(1+2 b /\left\|S_{N}\right\|\right)
\end{aligned}
$$

where $b \equiv \max \left\{\left\|S_{K-1}\right\|,\left\|S_{K}\right\|\right\}$.

Proof.

$$
\begin{aligned}
S_{L}=S_{N}+\left(S_{N+K}-S_{N}\right)+ & \left(S_{2 N+K}-S_{N+K}\right)-\left(S_{2 N+K}-S_{L}\right), \\
2(1-\tau(K+1))\left\|S_{N}\right\|^{2} & \leqslant\left\|S_{N}+S_{2 N+K}-S_{N+K}\right\|^{2} \\
& <2(1+\tau(K+1))\left\|S_{N}\right\|^{2}
\end{aligned}
$$

and Lemma 1 follows from Minkowski's inequality.

LEMMA 2. Given any $\varepsilon>0$ and any positive integer $L_{0}$, there are positive integers $N$ and $L$ with $L \geqslant L_{0}$ such that $\forall l, L \leqslant l \leqslant 2 L$, one has

$$
(1-\varepsilon) g(N) \leqslant g(l) \leqslant(1+\varepsilon) g(N) .
$$

Proof. For each integer $J \geqslant 2, \operatorname{Corr}\left(S_{N}, S_{J N}-S_{(J-1) N}\right) \rightarrow 0$ as $N \rightarrow \infty$. (For $J=2$ this just follows from (1).) Hence $\forall J>2, g(N J) / g(N) \rightarrow 1$ as $N \rightarrow \infty$. 
Let $M$ be a positive integer such that $(1-\varepsilon)<(1-\varepsilon)^{1 / 2}-M^{-1 / 2}<(1+\varepsilon)^{1 / 2}$ $+M^{-1 / 2}<(1+\varepsilon)$. Let $N$ be such that $M N \geqslant L_{0}$,

$$
\left\|S_{N}\right\|=\max \left\{\left\|S_{k}\right\|, 1<k \leqslant N\right\}
$$

and for each $m, M \leqslant m \leqslant 2 M$, one has

$$
(1-\varepsilon)^{1 / 2}<g(m N) / g(N) \leqslant(1+\varepsilon)^{1 / 2} .
$$

Let $L=M N$.

If $L \leqslant l \leqslant 2 L$, then for some $m$ one has $M \leqslant m<2 M-1$ and $m N<l<$ $(m+1) N$, and hence

$$
\begin{aligned}
(1-\varepsilon) g(N) & <\left[(1-\varepsilon)^{1 / 2}-(m+1)^{-1 / 2}\right] g(N) \\
& \leqslant[(m+1) N]^{-1 / 2}\left(\left\|S_{(m+1) N}\right\|-\left\|S_{N}\right\|\right) \\
& \leqslant g(l) \leqslant(m N)^{-1 / 2}\left(\left\|S_{m N}\right\|+\left\|S_{N}\right\|\right) \\
& \leqslant\left[(1+\varepsilon)^{1 / 2}+m^{-1 / 2}\right] g(N)<(1+\varepsilon) g(N)
\end{aligned}
$$

and Lemma 2 is proved.

Let $0<A<1$ be sufficiently small that if $\left(a_{n}, n=1,2, \ldots\right)$ is any sequence of real numbers such that $\Sigma_{n}\left|a_{n}\right| \leqslant A$ then $\left|1-\Pi_{n}\left(1+a_{n}\right)\right|<2 \Sigma_{n}\left|a_{n}\right|$ and $\mid 1-$ $\Pi_{n}\left(1+a_{n}\right)^{-1}\left|\leqslant 2 \Sigma_{n}\right| a_{n} \mid$. Let [] denote the greatest-integer function.

Proof of Theorem 1(i). Assume $0<\varepsilon<A$. Let $N$ be a positive integer such that $\sum_{n=0}^{\infty} \tau\left(\left[2^{N+n / 6}\right]\right)<\varepsilon / 6$. Let $C>0$ be such that $\sum_{n=0}^{\infty} 2^{N+1-n / 6} C^{-1}<\varepsilon / 6$. Let $L_{0}$ be a positive integer such that (i) $\forall J \geqslant L_{0},\left\|S_{J}\right\|>C$, (ii) $\sum_{n=0}^{\infty} 2^{-n} L_{0}^{-1}<$ $\varepsilon / 6$, and (iii) $\forall J \geqslant L_{0}, g(2 J)>2^{-1 / 6} g(J)$ and $g(2 J+1)>2^{-1 / 6} g(J)$ (see Lemma 1). For each $n=0,1,2, \ldots$ let $K_{n}=\left[2^{N+n / 6}\right]$. Using Lemma 2 , let the positive integers $H$ and $L$ be such that $L \geqslant L_{0}$ and $\forall l, L \leqslant l<2 L$, one has $(1-\varepsilon) g(H)$ $<g(l)<(1+\varepsilon) g(H)$.

Let $m$ be an arbitrary positive integer satisfying $m>2 L$. We wish to prove $(1-\varepsilon)^{2} g(H)<g(m)<(1+\varepsilon)^{2} g(H)$.

For some positive integer $M, 2^{M} L \leqslant m \leqslant 2^{M+1} L$. There is a sequence of positive integers $J_{0}, J_{1}, \ldots, J_{M}$ such that $m=J_{M}, L \leqslant J_{0} \leqslant 2 L$, and for each $n=$ $0,1, \ldots, M-1, J_{n+1}=2 J_{n}$ or $J_{n+1}=2 J_{n}+1$.

For each $n=0,1, \ldots, M-1$, one has $\left\|S_{J(n)}\right\| \geqslant 2^{n / 3}\left\|S_{J(0)}\right\| \geqslant 2^{n / 3} C$, and using Lemma 1 and the inequality $\left\|S_{K}\right\| \leqslant K$ one has

$$
\begin{aligned}
g\left(J_{n+1}\right) & \geqslant g\left(J_{n}\right)\left(2 J_{n} / J_{n+1}\right)^{1 / 2}\left(1-\tau\left(K_{n}+1\right)\right)^{1 / 2}\left(1-2 K_{n} /\left(2^{n / 3} C\right)\right) \\
& \geqslant g\left(J_{n}\right)\left(1-2^{-n} L_{0}^{-1}\right)^{1 / 2}\left(1-\tau\left(K_{n}+1\right)\right)^{1 / 2}\left(1-2^{N+1-n / 6} C^{-1}\right), \\
g\left(J_{n+1}\right) & \leqslant g\left(J_{n}\right)\left(1+\tau\left(K_{n}+1\right)\right)^{1 / 2}\left(1+2 K_{n} /\left(2^{n / 3} C\right)\right) \\
& \leqslant g\left(J_{n}\right)\left(1+\tau\left(K_{n}+1\right)\right)^{1 / 2}\left(1+2^{N+1-n / 6} C^{-1}\right) .
\end{aligned}
$$

Hence

$$
\begin{aligned}
& g(m) \geqslant g\left(J_{0}\right) \prod_{n=0}^{M-1}\left[\left(1-2^{-n} L_{0}^{-1}\right)^{1 / 2}\left(1-\tau\left(K_{n}+1\right)\right)^{1 / 2}\left(1-2^{N+1-n / 6} C^{-1}\right)\right], \\
& g(m) \leqslant g\left(J_{0}\right) \prod_{n=0}^{M-1}\left[\left(1+\tau\left(K_{n}+1\right)\right)^{1 / 2}\left(1+2^{N+1-n / 6} C^{-1}\right)\right] .
\end{aligned}
$$


Since $\varepsilon<A$ we get $(1-\varepsilon) g\left(J_{0}\right) \leqslant g(m) \leqslant(1+\varepsilon) g\left(J_{0}\right)$ and hence $(1-\varepsilon)^{2} g(H)<$ $g(m)<(1+\varepsilon)^{2} g(H)$, which is what we wanted to prove.

Hence Lim inf $g(n)$ and Lim sup $g(n)$ are finite positive numbers, and their ratio can be forced arbitrarily close to 1 if $\varepsilon$ is chosen sufficiently small. Theorem 1(i) follows.

Proof of Theorem 1(ii). Let $\sigma^{2}$ be as in Theorem 1(i). Assume $0<\gamma<1$. There are constants $0<C_{1}<C_{2}$ such that, for all $n=1,2,3, \ldots, C_{1}<g(n)<C_{2}$.

Let $N$ be a positive integer such that $\sum_{n=0}^{\infty} \tau\left(\left[2^{(N+n) \gamma}\right]\right)<A / 3$. Let $L$ be a positive integer such that $\sum_{n=0}^{\infty} 2^{1+N+n(\gamma-1) / 2} C_{2} /\left(C_{1} L\right)<A / 3$.

Suppose $M$ is a nonnegative integer and $2^{M} L^{2}<m<2^{M+1} L^{2}$. For each $n=$ $0,1,2, \ldots$ let $J_{n}=2^{n} m$ and let $K_{n}=\left[2^{(N+n) \gamma}\right]$. Then by Lemma 1 ,

$$
\begin{aligned}
g\left(J_{n+1}\right) & \geqslant g\left(J_{n}\right)\left(1-\tau\left(K_{n+M}+1\right)\right)^{1 / 2}\left(1-2 C_{2} K_{n+M}^{1 / 2} /\left(C_{1} J_{n}^{1 / 2}\right)\right) \\
& \geqslant g\left(J_{n}\right)\left(1-\tau\left(K_{n+M}+1\right)\right)^{1 / 2}\left(1-C_{2}\left(C_{1} L\right)^{-1} 2^{1+N_{\gamma} / 2+(M+n)(\gamma-1) / 2}\right), \\
g\left(J_{n+1}\right) & \leqslant g\left(J_{n}\right)\left(1+\tau\left(K_{n+M}+1\right)\right)^{1 / 2}\left(1+2 C_{2} K_{n+M}^{1 / 2} /\left(C_{1} J_{n}^{1 / 2}\right)\right) \\
& \leqslant g\left(J_{n}\right)\left(1+\tau\left(K_{n+M}+1\right)\right)^{1 / 2}\left(1+C_{2}\left(C_{1} L\right)^{-1} 2^{1+N_{\gamma} / 2+(M+n)(\gamma-1) / 2}\right) .
\end{aligned}
$$

Since $\operatorname{Lim}_{l \rightarrow \infty} g(l)=\sigma$ one has

$$
\begin{aligned}
& g^{2}(m) \leqslant \sigma^{2} \cdot \prod_{n=0}^{\infty}\left[\left(1-\tau\left(K_{n+M}+1\right)\right)^{-1}\left(1-C_{2}\left(C_{1} L\right)^{-1} 2^{1+N \gamma / 2+(M+n)(\gamma-1) / 2}\right)^{-2}\right], \\
& g^{2}(m) \geqslant \sigma^{2} \cdot \prod_{n=0}^{\infty}\left[\left(1+\tau\left(K_{n+M}+1\right)\right)^{-1}\left(1+C_{2}\left(C_{1} L\right)^{-1} 2^{1+N \gamma / 2+(M+n)(\gamma-1) / 2}\right)^{-2}\right],
\end{aligned}
$$

and hence by the definition of $A$,

$$
\left|\sigma^{2}-g^{2}(m)\right| \leqslant 2 \sigma^{2}\left[\sum_{n=0}^{\infty} \tau\left(K_{n+M}+1\right)+2 \sum_{n=0}^{\infty} 2^{1+N \gamma / 2+(M+n)(\gamma-1) / 2} C_{2} /\left(C_{1} L\right)\right] \text {. }
$$

If we let $M \rightarrow \infty$ and $m \rightarrow \infty$ subject to the restriction $2^{M} L^{2}<m<2^{M+1} L^{2}$, then we get the following:

$$
\begin{aligned}
& \sum_{n=0}^{\infty} 2^{(M+n)(\gamma-1) / 2}=O\left(2^{M(\gamma-1) / 2}\right)=O\left(m^{(\gamma-1) / 2}\right), \\
& \sum_{n=0}^{\infty} \tau\left(K_{n+M}+1\right) \leqslant \sum_{n=0}^{\infty} \tau\left(\left[2^{n \gamma} m^{\gamma} / L^{2}\right]+1\right) .
\end{aligned}
$$

Theorem 1(ii) follows.

ACKNOWLEDGMENTS. The author thanks the referee and the editor for their careful reading of the manuscript and their helpful suggestions.

\section{REFERENCES}

1. I. Berkes and W. Philipp, Approximation theorems for independent and weakly dependent random vectors, Ann. Probab. 7 (1979), 29-54.

2. I. A. Ibragimov, A note on the central limit theorem for dependent random variables, Theory Probab. Appl. 20 (1975), 135-141.

3. I. A. Ibragimov and Y. A. Rozanov, Gaussian random processes, Applications of Math., no. 9, Springer-Verlag, New York, 1978.

4. ___ Gaussian random processes, "Nauka", Moscow, 1970. (Russian)

Department of Mathematics, Indiana University, BloOmmgton, Indiana 47405 